\title{
Dual use of LED traffic signal system
}

\author{
Y. Y. Tam*, C. W. Man, D. Yang, S. W. Cheung, E. S. Yang \\ Department of Electrical and Electronic Engineering \\ The University of Hong Kong
}

\begin{abstract}
The dual use, signaling and communication, of LED traffic signal system is described and analyzed. The primary function of a traffic light system is to give traffic and pedestrian signals. A prototype of LED traffic signal head is developed to perform a secondary function: communication. A wireless communication link is set up using the LED traffic signal head as the transmitter. The LEDs are modulated to transmit information-carrying light. The receiver uses a silicon photodiode to detect the transmitted radiation. Using visible light as the transmission medium, a $1 \mathrm{Mbit} / \mathrm{s}$ wireless data link is obtained.
\end{abstract}

Keywords: LED, traffic light, wireless communication

\section{INTRODUCTION}

The performance, in terms of luminous efficiency, of visible light-emitting diodes (LED) has been improved tremendously over the past ten years ${ }^{1-4}$. Red-emitting double-heterostructure (DH) AlGaAs devices can now offer comparable performance as incandescent lamps. The newer red-orange and amber AlInGaP devices are even better. Figure 1 shows the evolution of visible light-emitting diode (LED) performance with time. The performances of filtered and unfiltered incandescent lamps are also included for comparison.

The luminous performance is measured in lumens per watt $(\mathrm{lm} / \mathrm{W})$. It is the ratio of luminous (visible) flux output of a light source measured in lumens divided by the electrical power input to the device. Lumens are calculated by multiplying the radiant flux output of a device (in watts) by the human eye's sensitivity as defined by the Commission Internationale de L'Eclairage (CIE) ${ }^{16}$. A typical $100 \mathrm{~W}$ incandescent lamp produces about $15 \mathrm{~lm} / \mathrm{W}$ of white light. But after red-filtering, about $75 \%$ of the light is absorbed and only $3-4 \mathrm{~lm} / \mathrm{W}$ of red light is left. A commercially available red AlGaAs LED $(650 \mathrm{~nm})$ can give $8 \mathrm{~lm} / \mathrm{W}$ of red light, while a red-orange AlInGaP device $(629 \mathrm{~nm})$ can achieve a better luminous performance of $30 \mathrm{~lm} / \mathrm{W}$ but at shorter wavelength. Table 1 lists the characteristics of some commercially available LEDs ${ }^{5-6}$.

In the past, blue was the missing color in the visible spectrum that could be provided by LEDs and green LED was never efficient enough to attain high brightness. With the recent introduction of InGaN device, high efficiency blue and green LEDs become achievable. The whole color spectrum is now under a better coverage. Figure 2 shows the luminous efficiencies of LEDs plotted against wavelength. The eye response curve (CIE) is included for reference. AlGaAs LED is a red-only device; while AlInGaP LED covers the red-orange to yellow-green spectrum. InGaN devices cover yellow-green to blue region. White light can be obtained by appropriately mixing LEDs of different colors.

Using high brightness LEDs, a wide variety of new applications emerge ${ }^{7}$. These include the traffic signal light, the automotive signal light, the variable message sign (VMS) on highway, the light crystal display (LCD) backlighting and the large-area display, etc. All these applications consume a large number of visible LEDs, making it at present the largest compound semiconductor device type in terms of sales volume.

In this paper, we take a special interest on the LED traffic signal system. Some cities in US and Europe have already started the development of LED traffic signal system a few years ago ${ }^{8.14}$. After a series of laboratory and on-street testing, performance specifications have been drafted. These specifications address issues such as initial brightness, lensing requirements, physical compatibility, allowable long-term degradation, power factor, maximum power, harmonic distortion and night dimming. Luminous distribution and chromaticity requirements generally follow current and anticipated requirements of the Institute of Transportation Engineers (ITE), although that agency is still in the process of adopting standards for LED signals.

* Correspondence: Email: yytamäajee.hku.hk; Telephone: (852) 2857 8409; Fax (852) 25598738 
The major benefit of replacing the existing incandescent traffic signal with the LED ones is a reduction in power consumption ${ }^{8}$. The standard 12" traffic light currently in use utilizes a $150 \mathrm{~W}$ incandescent lamp. Whereas a 12 " LED traffic light, on the other hand, consumes just $25 \mathrm{~W}$. For a red signal with a $60 \%$ duty cycle and an energy cost of US\$ 0.1 per $\mathrm{kWh}$, the annual energy savings is more than US $\$ 60$ per head. If a city has 10,000 heads, the annual savings would be more than US $\$ 600,000$. The cumulative energy costs savings after 7 years of operation (which is the typical lifetime of LED signals) will be US $\$ 4,200,000$. The initial cost of installing the LED traffic signals will be paid back in the long run.

The maintenance cost is also greatly reduced. In general, most municipalities re-lamp all red and blue-green traffic signals annually and yellow traffic signals every two years. Also, many transit agencies require the immediate replacement of failed red traffic signals and replacement of failed yellow and blue-green traffic signals the next business day in order to minimize the municipality's liability. The longer life expectancy of LED (7 years of lifetime) not only cut down the equipment maintenance cost, but also the human power cost. The primary safety concerns of red lamp burnout is also minimized, which helps to reduce the possibility of traffic accident.

Since the use of LED traffic signals is coming, here we further explore the usefulness of LED by making it to serve dual purpose: signaling and communication. The light given out from the traffic signal head is originally intended for human vision (signaling purpose). It gives a red signal for "stop" and green signal for "go". The drive current for the LED may be either DC-constant or low-frequency modulated (less than $1 \mathrm{kHz}$ ) with a certain duty cycle. However, with the use of LEDs, it is possible to modulate the light with a much higher frequency. If the emitted light is encoded with data, the LED signal head can be used for communication purpose and that is the objective of this study.

In the following sections, we will first discuss some important properties of high brightness visible LED. The dual use of LEDs will be described. An experimental wireless communication link using LED traffic signal head will be discussed and analyzed.

\section{IMPORTANT PROPERTIES OF LED FOR DUAL USE TRAFFIC SIGNAL}

The luminous performance of LED can be estimated by the following equation ${ }^{5}$ :

$$
\text { luminous performance } \cong 683 \mathrm{~V}(\lambda) \eta_{i m t} C_{e x}
$$

where $\lambda$ is the wavelength of the emitter, $683 \mathrm{~V}(\lambda)$ is the CIE value (given by the CIE eye response curve) ${ }^{16}, \eta_{\text {int }}$ is the internal quantum efficiency and $C_{e x}$ is the extraction efficiency.

The best commercially available device by now is red-orange AlInGaP LED, with luminous performance over $30 \mathrm{~lm} / \mathrm{W}$. The internal quantum efficiency is approaching $100 \%$; while the extraction efficiency is about $30 \%$.

Reliability is another reason why LED is such an attractive alternative to incandescent lamp. Under normal operating condition, LED seldom blows out. Instead, it degrades gradually over time. High temperature operating life (HTOL) testing is used to project long term light output degradation ${ }^{10}$. The test is performed in an ambient of $+55^{\circ} \mathrm{C}$ with $20 \mathrm{~mA} \mathrm{DC}$ forward current. The LED light output decreases by $-13.5 \%$ after 16,000 hour continuous operation. The average long term light output degradation at 100,000 hour is projected to be about $-27 \%$. This means that at normal operating temperature and current, a LED can typically serve for several years of operation. This reliable performance is much superior to that of incandescent lamp, which has a life time of only about 1,000 hour ". The color of the emitted light also does not change during the life time of the LED.

Another important characteristics of LED is the fast response time. A fast LED can be turned on or off on the order of tens nanoseconds. This rapid switching capability can hardly be found in other types of light sources. LED can be operated with either dc or pulsed drive current. If the strobing frequency of the pulsed drive current is over $100 \mathrm{~Hz}$, human eye will see stable light output without observable flickers.

Since the response time of the LED is so fast, we can increase the strobing frequency up to a much high frequency, with the LED still being able to be completely turned on and off. At this frequency range, the emitted light can also be used for data communication purpose. Instead of using a simple pulsed drive current, we modulate the LED to transmit information- 
carrying light. A wireless communication link can be set up using visible light as the transmission medium. A LED (or group of LEDs) is used as the transmitter. The receiver side uses a silicon photodiode to detect the transmitted light and generate corresponding photocurrent.

This brings out the dual use of LED: signaling and communication. Other light sources may not be suitable for these dual functions. For example, incandescent lamps and fluorescent lamps cannot be switched on and off quickly. Laser diode and infrared LED can be high frequency modulated, but the laser light is dangerous for direct vision with human eye and our eyes cannot see infrared radiation. So, this dual use characteristic is quite unique to visible LED.

Two of the main advantages of using LED are that 1) the visible light spectrum us unregulated world wide and so we can freely use the spectrum, and 2) the receivers is very cheap to implemented.

\section{OTHER POSSIBLE APPLICATIONS WITH THE DUAL USE OF LED}

In this paper, although we focus on the dual use of LED traffic signal systems, the same concept may well be used in many other applications such as the followings.

\section{a) Automotive signal light}

Using LED for automotive signal light has many advantages over using incandescent lamps. LED has a much longer life expectancy which helps to reduce the possibility of blowing out. LED consumes less power and generates less heat. The color of the emitted light is determined by the type of LED used, rather than the color filter.

An automotive LED signal light can be used for signaling as well as communications at the same time. Therefore, together with the LED traffic signal system mentioned before, a full-duplex wireless communication link can be set up between the traffic light system and the vehicle and information can be passed between them. For vehicles using LEDs as the signal lights, then inter-vehicle communication is also possible.

\section{b) Variable message sign}

Variable message sign (VMS) is becoming a popular and important element on highways. VMS is a useful tool to remind drivers of the current road conditions, such as speed limitation, traffic jam or traffic redirection due to road work, and to give warnings in case of accident or emergency in the road ahead. If the VMS is made of LEDs, then at the same time, the dual use of LEDs can enable the VMS to broadcast, in form of audio signal, the detail of the event.

\section{c) Large-area LED display}

The VMS just mentioned above is an example of using large-area LED displays. Other applications of using large LED displays include the information systems at airports, bus and railway stations, the arena scoreboards, the advertising signs, etc. In these applications, the LED displays are used to provide information to interested people. If the LEDs are dually used, extra information can be provided through the modulation of the light transmitted by the LEDs. A portable optical receiver can be used to demodulate the received light and recover the information. Since the amount of displayed information is also limited by the size of the display, through the dual use of LEDs, extra information can be delivered.

\section{d) Electronic toll collection system}

The existing electronic toll collection systems use microwave frequency as the communication links for the system to communicate with the passing vehicles. Microwave methods require the costly installation of communication infrastructure. However, since many signaling elements on the roads will be made of LEDs, e.g. the traffic light, the "STOP" sign, the "EXIT" sign, the automotive light and VMS, if these LED elements are used as the communication interfaces of the electronic toll collection systems, then the extra installation costs of the systems will be much reduced. 


\section{e) Wireless local area network}

Wireless local area network (LAN) is usually implemented using microwave or infrared light. Visible light may be a possible alternative. With the advance of $\mathrm{GaN}$ based devices, high brightness white LED is now achievable. The luminous performance is high enough so that we can construct a LED lamp (consisting of an array of white LEDs) for indoor illumination purpose. Alternatively, white LED lamp can also be built by appropriately mixing LEDs of different colors. A LED lamp mounted on the ceiling can be used as the host of the network. The remote terminals can be placed any laminated areas of the room. Here, the indoor communication network is realized using visible light spectrum and the visible light serves dual purposes: illumination and communication.

\section{EXPERIMENTAL WIRELESS LINK SETUP USING LED TRAFFIC SIGNAL HEAD}

To investigate the dual use of LEDs, we have set up an experimental 1-Mbit/s wireless communication link using a prototype of LED traffic signal head and a receiver.

\section{a) Prototype of LED traffic signal head}

Our prototype of LED traffic signal head uses commercially available AlInGaP LED 9 . This LED emits red light with dominant wavelength of $626 \mathrm{~nm}$ and spectral halfwidth of $17 \mathrm{~nm}$. The viewing angle is $60^{\circ}$. It gives a luminous intensity of $180 \mathrm{mcd}$ at $20 \mathrm{~mA}$ forward current. Due to the wide viewing angle, the emitted light is more spread out and the luminous intensity is thus lower.

Figure 3 shows the measured radiation pattern of the LED. The graph is plotted with normalized intensity (in decibel) against angular displacement. The measured data conforms to the specifications of the LED. The normalized intensity drops by $3-\mathrm{dB}$ at $30^{\circ}$ point. Also, within $20^{\circ}$ on both sides of the axis, the normalized intensity varies by less than $1-\mathrm{dB}$. Figure 4 shows the rectangular pulse response graph of the LED. A $1 \mu$ s rectangular pulse was used to intensity modulate the LED. Both turn-on and turn-off time of the LED were found to be less than $50 \mathrm{~ns}$.

We use a current limiting resistor to connect in series with the LED so that a $30 \mathrm{~mA}$ DC current flows through the LED in idle state. When the LED is in transmitting state, the LED is turned on to transmit a data bit of ' 1 ' and off to transmit a ' 0 '. The average current flowing through the LED will be equal to $15 \mathrm{~mA}$, assuming that the number of ' 1 ' is the same as that of ' 0 '. This assumption is generally true, especially when the amount of data to be transmitted is very large. This LED drive current is analogous to a pulsed drive current with $50 \%$ duty cycle, since the LEDs are $50 \%$ of the time on and $50 \%$ of the time off.

In our prototype, we have used a total of 64 LEDs. All LEDs are modulated with the same drive current at the same time. Thus, the transmitting power is 64 times that of a single LED. Figure 5 shows the measured radiation pattern of the prototype. The $3-\mathrm{dB}$ beamwidth is about $30^{\circ}$, which is the same as that of a single LED.

The LED signal head acts as the transmitter of the wireless communication link. In order to simulate data traffic, a pseudorandom pattern generator, having $2^{16}-1$ different bit patterns, is used to generate the random data. The LEDs are intensity-modulated to transmit the data. The transmitted signal uses on-off keying modulation (OOK).

\section{b) Receiver}

A $15 \mathrm{~mm}^{2}$ silicon photodiode is used to detect the transmitted light and converts it into photocurrent. The photodiode is coupled to a low noise transimpedance amplifier to convert the photocurrent into voltage signal and amplify it. Another amplifier block follows to provide higher gain value. The amplified signal then passes through a matched filter, which is implemented by an integrate-and-dump circuit. The filtered signal is digitized into either bit ' 1 ' or ' 0 ' with a quantizer. The original data is recovered. Figure 6 shows the received and amplified waveform at the input of the matched filter. 


\section{EXPERIMENTS, RESULTS AND DISCUSSIONS}

Using the wireless communication link described above, different experiments have been carried out to investigate the system performance.

\section{a) Optical path loss experiment}

Theoretically, the received power is inversely proportional to the square of the distance between the transmitter and the receiver, i.e.:

$$
\operatorname{Pr} \propto \frac{1}{4 \pi R^{2}}
$$

where $P_{r}$ is the received power and $R$ is the distance between the transmitter and receiver. This equation indicates that if the received power is plotted against the distance $R$, the graph will be a straight line having a slope of $-20 \mathrm{~dB} / \mathrm{decade}$. In other words, the received power decreases by $20 \mathrm{~dB}$ when the distance is increased by 10 times.

Figure 7 shows the measured results of received power intensity plotted against distance. An optical power meter was used to measure the received power from the LED traffic signal head. The graph shows a straight line with a slope of about -20 $\mathrm{dB} / \mathrm{decade}$. Thus the experimental results match with the theoretical calculation and so this confirms that equation 2 can be used in link budget calculation of the wireless visible light communication link.

\section{b) Bit-error rate experiment}

The bit-error rate (BER) of the wireless communication link was measured. In this experiment, the ambient light is considered as interference to the wireless visible light communication link. Different types of ambient radiation degrade the system performance at different extents. In order to identify each type of radiation and investigate the effects, the experiments were carried out in a closed room with no window. The BER measurements were then carried out under different conditions: in the absence of any other ambient light, in the presence of incandescent light and in the presence of fluorescent light.

Figure 8 shows the BER measurement results against the average received irradiance $\left(\mathrm{dBm} / \mathrm{cm}^{2}\right)$ in the absence of ambient light. It can be seen that a BER of $10^{-6}$ is achieved with received irradiance of $-26.8 \mathrm{dBm} / \mathrm{cm}^{2}$.

Since many vehicles still use the incandescent lamps for head light and tail light, it is worthwhile to study the effect of incandescent light on the BER performance of the system. In the closed room, an incandescent lamp was used to produce an interference level of $3.80 \mu \mathrm{W} / \mathrm{cm}^{2}\left(-24.2 \mathrm{dBm} / \mathrm{cm}^{2}\right)$ at the receiver. The BER of the system were measured with different values of received irradiance as shown in Fig. 9. In the presence of incandescent light, the system achieves a BER of $10^{-6}$ at $-26.6 \mathrm{dBm} / \mathrm{cm}^{2}$. Therefore now the system requires $0.2 \mathrm{~dB}$ more power to achieve the same performance than the system with no incandescent light as interference.

The effect of fluorescent light on the BER performance of the system was also studied. Although most vehicles do not use fluorescent lamps, many advertisement signs on the roadsides use them. In the closed room, a fluorescent lamp was used to produce an interference level of $1.90 \mu \mathrm{W} / \mathrm{cm}^{2}\left(-27.2 \mathrm{dBm} / \mathrm{cm}^{2}\right)$ at the receiver. The BER of the system for different values of received irradiance were measured. Then more fluorescent lamps were used to increase the interference level to 3.80 $\mu \mathrm{W} / \mathrm{cm}^{2}\left(-24.2 \mathrm{dBm} / \mathrm{cm}^{2}\right)$. The BER measurement was repeated. Figure 10 shows the measurement results. In the presence of $1.9 \mu \mathrm{W} / \mathrm{cm}^{2}$ fluorescent light, a BER of $10^{-6}$ is achieved with a received irradiance of $-25.8 \mathrm{dBm} / \mathrm{cm}^{2}$. This means that 1 $\mathrm{dB}$ more power is required relative to the condition without any fluorescent light. When the fluorescent light intensity is increased to $3.8 \mu \mathrm{W} / \mathrm{cm}^{2}$, the received irradiance required to achieve again the same BER of $10^{-6}$ is $-24.2 \mathrm{dBm} / \mathrm{cm}^{2}$. Thus a $2.6 \mathrm{~dB}$ more power is required to achieve the same performance, relative to the condition with no fluorescent light.

It can be seen from Fig. 9 and Fig. 10 that performance degradation due to fluorescent light is larger than that of incandescent light. This is because the light emitted from the incandescent lamp is relatively constant. The performance degradation is caused by the increased shot noise generated in the photodiode. While the fluorescent lamp emits cycle- 
stationary low frequency lights which are varying in time. Thus at the same interference level, the fluorescent light has greater effects on system performance than does the incandescent light.

\section{c) Field test}

In the indoor environments, each type of ambient lights as the source of interference can be controlled carefully and measured precisely so that its effect on the system performance can be studied. However, in the outdoor environment, there many different types of interference which are very difficult to control and so the studies of the system in the outdoor environments become impossible. Nevertheless, we have carried out field tests to demonstrate the dual use LED traffic signal system. A wireless communication link was set up using an LED traffic signal head and a corresponding receiver. The LED traffic signal head was used to broadcast several multiplexed signals, including digitized voice signals and digital data signals. To increase the received power, a small lens was used at the receiver to collect the transmitted light. The LED traffic signal head and the receiver were placed at a distance of well over 100 feet. We found that the receiver was able to demodulate and reproduce the voice signals and recover the digital data. Other forms of digital data such as image data have also been tried and the results were satisfactory.

\section{d) Correlation with photometric values}

To illustrate the BER performance of a wireless optical communication system, irradiance is usually used as the measurement unit. It is a radiometric unit, given by the value of radiant flux per unit area, i.e.

$$
\text { irradiance }=\frac{\text { radiant flux }}{\text { area }}
$$

The measurement unit used for radiant flux is watt (W) and the unit for irradiance is $\mathrm{W} / \mathrm{m}^{2}$. However, if we want to investigate human visual response, we have to deal with photometric values instead of radiometric values. Since our proposed LED traffic signal system is intended to serve dual purposes (signaling and communication), it is worthwhile to do some calculation to see the correlation between radiometric values and photometric values.

The unit of luminous flux is called a lumen (lm). With given value of radiant flux, the luminous flux can be calculated by the following equation ${ }^{12}$ :

$$
\phi_{v}=683 \operatorname{lm} / W \int \phi_{e}(\lambda) V^{\prime}(\lambda) d \lambda
$$

where $\phi_{v}$ is the luminous flux, $\phi_{e}(\lambda)$ is the radiant spectrum of the radiation source, and $V^{\prime}(\lambda)$ is the CIE luminosity function (which is the same as the eye response curve as shown in Fig. 2). It is adopted by CIE as a human eye weighting factor, based on extensive testing on human subjects. The highest human eye sensitivity occurs at $555 \mathrm{~nm}$ and has a radiant flux to luminous flux conversion factor of $683 \mathrm{~lm} / \mathrm{W}$.

Illuminance is the photometric counterpart of irradiance. It is given by the value of luminous flux per unit area, i.e.

$$
\text { illuminance }=\frac{\text { luminous flux }}{\text { area }}
$$

The measurement unit for illuminance is $\operatorname{lux}\left(\mathrm{lm} / \mathrm{m}^{2}\right)$ or $\mathrm{f}_{\mathrm{c}}\left(\mathrm{lm} / \mathrm{ft}^{2}\right)$. In Fig. 8, the top axis uses the received illuminance $\left(\mathrm{lm} / \mathrm{m}^{2}\right)$ as the scale. It is converted from the received irradiance (the bottom axis) using equations 4 and 5 . From the graph, it can be noted that a BER of $10^{-6}$ is achieved with a received illuminance of 3.24 lux.

Knowing this illuminance value alone is of no special significance. It will be more useful if we compare this value with other ambient lighting illuminance levels. Table 2 shows some typical values of indoor ambient light levels ${ }^{15}$. An ambient illuminance level below 750 lux is considered as dim; while a level above 2000 lux is bright. In our BER experiment, the required illuminance level to achieve a BER of $10^{-6}$ is 3.24 lux, which is a pretty low value. 


\section{CONCLUSIONS}

In this paper, the dual use of LED traffic signal systems and other possible applications with the dual use of LED have been discussed. An experimental prototype of an LED traffic signal system using commercially available LEDs for dual use has been developed and tested. Different measurements have been take with the systems at a data rate of $1 \mathrm{Mbit} / \mathrm{s}$ in the indoor environments. Results have shown that a BER of $10^{-6}$ can be achieved with a received irradiance of $-26.8 \mathrm{dBm} / \mathrm{cm}^{2}$, which is equivalent to 3.24 lux. Different field tests have also been carried in the outdoor environments and results have shown that a reliable communication link can be attained over a distance of 100 feet.

\section{REFERENCE}

1. M. G. Craford, and F. M. Sternaka, "Light-Emitting Diodes", Encyclopedia of Applied Physics, G. L. Trigg, vol. 8, VCH Publishers, New York, 1994.

2. M. G. Craford, "LEDs challenge the incandescents," IEEE Circuits \& Devices Magazine, vol. 8, pp. 24-29, 1992.

3. M. G. Craford, "LEDs get brighter ... much brighter", Information Display, vol. 9, pp. 12-14, 1993.

4. K. Werner, "Higher visibility for LEDs", IEEE Spectrum, vol. 31, pp. 30-39, 1994.

5. M. G. Craford, "Overview of device issues in high-brightness light-emitting diodes", High Brightness Light Emitting Diodes, G. B. Stringfellow and M. G. Craford, vol. 48, Academic Press, New York, 1997.

6. S. P. Denbaars, "Gallium-Nitride-based materials for blue to ultraviolet optoelectronics devices", Proceedings of the IEEE, vol. 85, pp. 1740-1749, 1997.

7. M. W. Hodapp, "Applications for high-brightness light-emitting diodes", High Brightness Light Emitting Diodes, G. B. Stringfellow and M. G. Craford, vol. 48, Academic Press, New York, 1997.

8. J. M. O'Connell, "Five years of LED traffic signal development", Traffic Technology International, Aug/Sept, pp. 106-110, 1997.

9. Hewlett-Packard Co., Optoelectronics Designer's Catalog, 1997.

10. Hewlett-Packard Co., "Projected long term HTOL light output degradation of precision optical performance AlInGaP LEDs", Application Brief I-018, 1998.

11. S. Peralta, and H. Ruda, "Applications for advanced solid-state lamps", IEEE Industry Applications Magazine, vol. 4, pp. 31-42, 1998.

12. S. Gage, D. Evans, M. Hodapp, and H. Sorensen, Optoelectronics Applications Manual, McGraw-Hill, New York, 1977.

13. Hewlett-Packard Co., "Pulsed operating ranges for AlInGaP LEDs vs. projected long term light output performance", Application Brief I-024, 1998.

14. D. Ibrahim, and M. Beasley, "The benefits of LED traffic lights in London and the pilot test sites", the $9^{\text {th }}$ International Conference on Road Transport Information and Control (Conf. Publ. No. 454), pp. 172-176, London, 1998.

15. Hewlett-Packard Co., "Contrast enhancement techniques for LED displays", Application Note 1015, 1997.

16. Commission Internationale de L'Eclairage, "Light as a true visual quantity: principles of measurement", $C I E$ publication number 41, 1978. 


\begin{tabular}{|ccccc|}
\hline Material & Color & $\begin{array}{c}\text { Peak } \\
\text { Wavelength } \\
(\mathrm{nm})\end{array}$ & $\begin{array}{c}\text { Luminous } \\
\text { Efficiency at 20mA } \\
(\mathrm{m} / \mathrm{W})\end{array}$ & $\begin{array}{c}\text { External Quantum } \\
\text { Effciency at 20mA } \\
(\%)\end{array}$ \\
\hline AlGaAs & Infrared & $780-880$ & $\mathrm{NA}$ & 27.0 \\
AlGaAs & Red & 650 & 9.0 & 20.0 \\
AlInGaP & Red-orange & 629 & 29.4 & 17.6 \\
AlInGaP & Orange & 604 & 41.5 & 11.5 \\
InGaN & Yellow & 590 & 4.0 & 1.2 \\
InGaN & Green & 550 & 25.0 & 5.0 \\
InGaN & Blue & 470 & 6.0 & 10.0 \\
\hline
\end{tabular}

Table 1 Characteristics of some commercially available visible LEDs

\begin{tabular}{|c|c|}
\hline Indoor incident ambient light & Lux $\left(\mathrm{lm} / \mathrm{m}^{2}\right)$ \\
\hline Dim & $250-750$ \\
Moderate & $750-2000$ \\
Bright & $2000-10000$ \\
\hline
\end{tabular}

Table 2 Typical indoor ambient illuminance levels

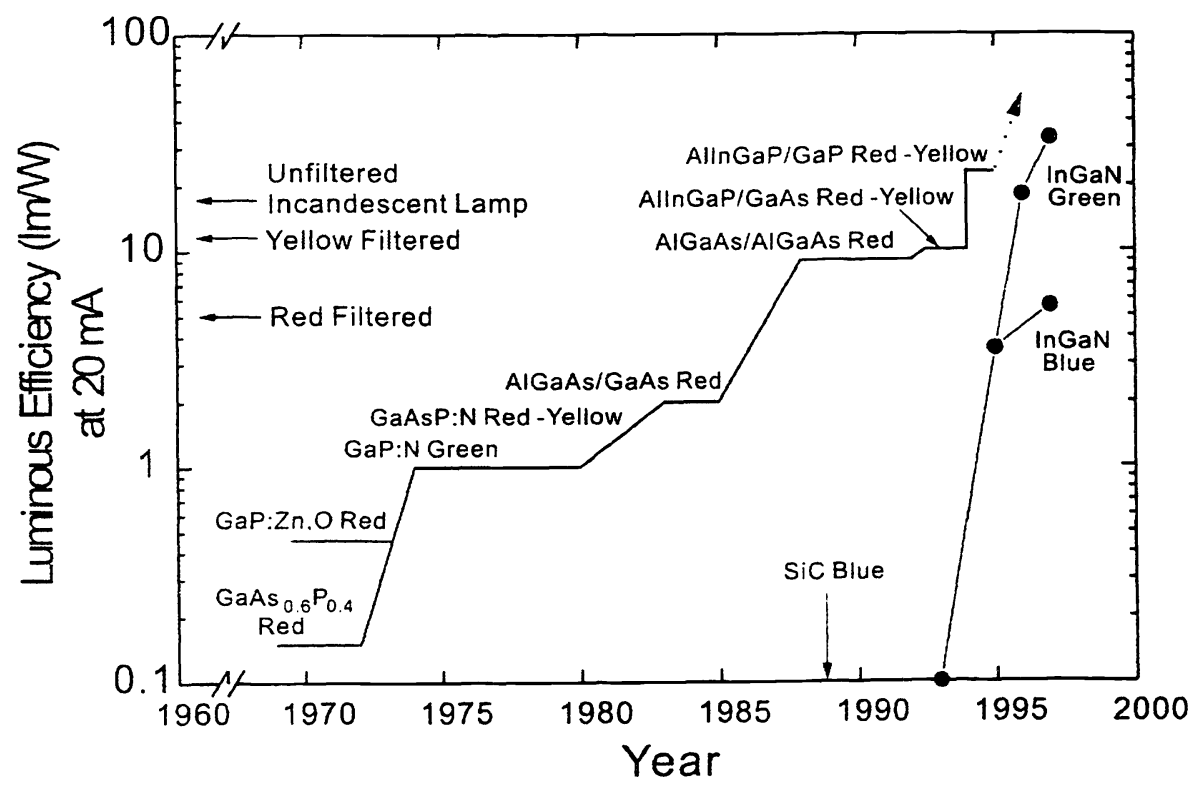

Fig. 1 Evolution of visible LED technology and performance. Luminous efficiencies of unfiltered and filtered incandescent lamps are included for comparison. 


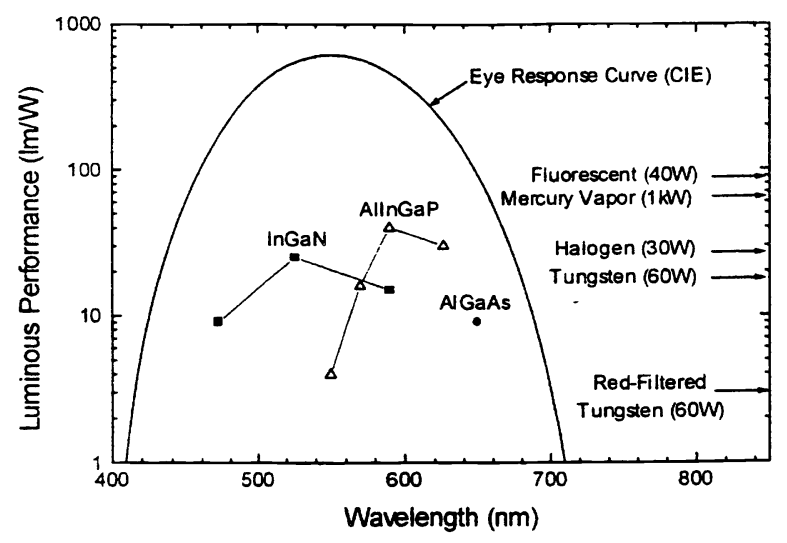

Fig. 2 Luminous Performance of visible LEDs plotted against wavelength. The performance of other light sources and the eye response curve (CIE) are included for reference.

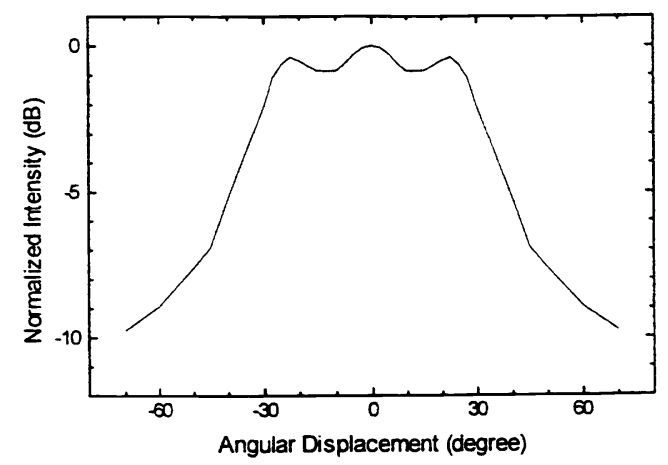

Fig. 3 Measured radiation pattern of a single LED. The peak value is normalized to $0 \mathrm{~dB}$.

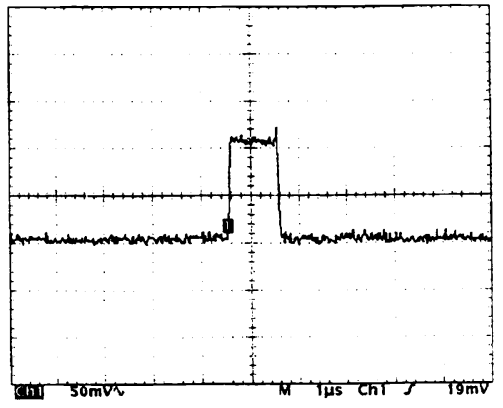

Fig. 4 Rectangular pulse response of the LED. The LED is intensity-modulated by a $1 \mu$ s rectangular pulse. The turn-on and turn-off time are both found to be $50 \mathrm{~ns}$. 


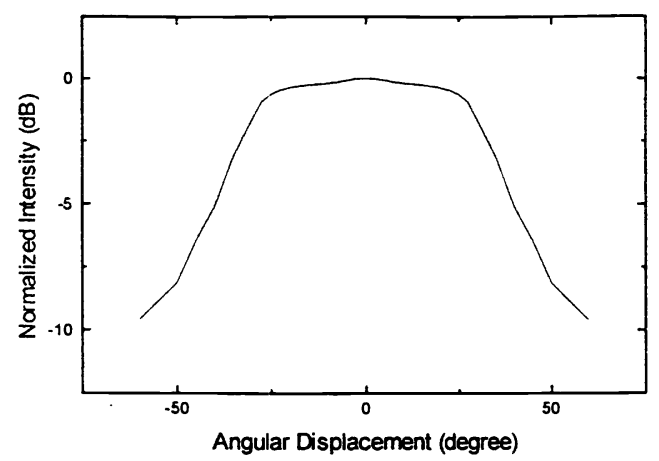

Fig. 5 Measured radiation pattern for the LED traffic signal head prototype. The peak value is normalized to $0 \mathrm{~dB}$.

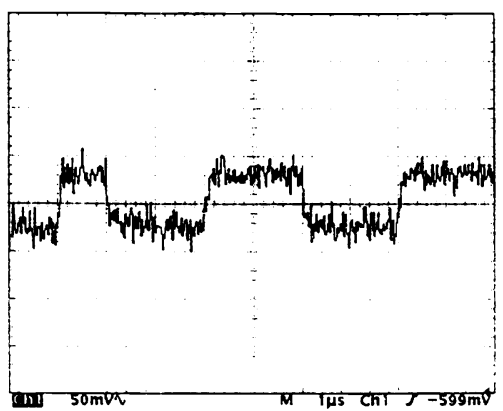

Fig. 6 Received and amplified signal at the input of the matched filter.

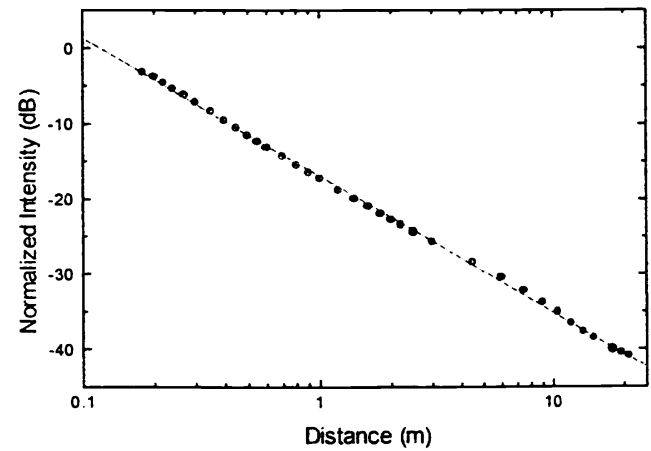

Fig. 7 Received power intensity (normalized) plotted against the distance between transmitter and receiver. 


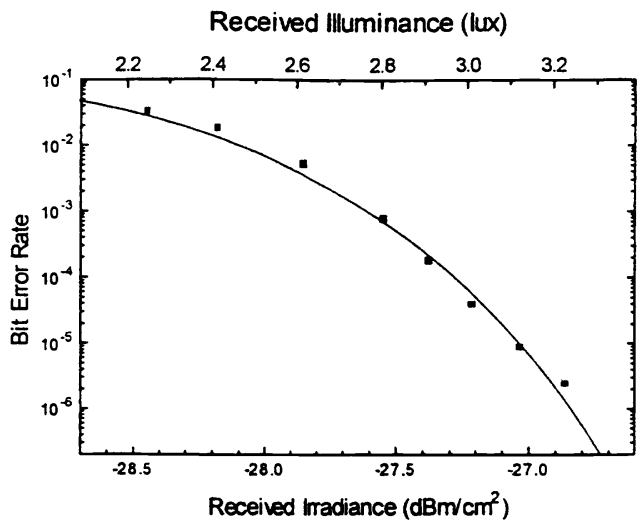

Fig. 8 BER performance of the LED traffic signal system. The bottom axis uses radiometric unit (irradiance) as the measurement scale; while the top axis uses photometric unit (illuminance).

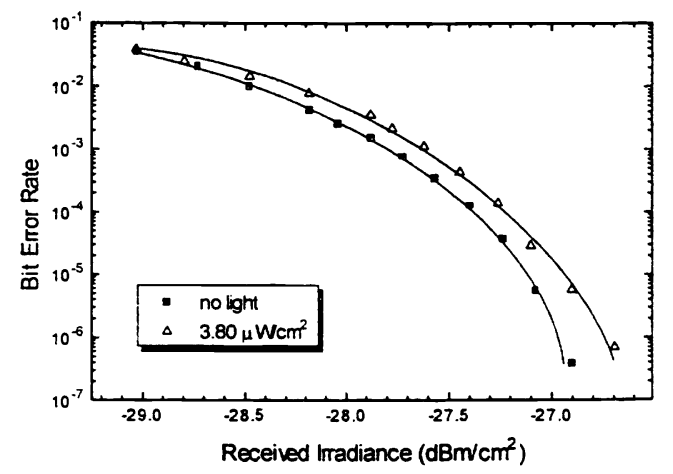

Fig. 9 BER performance of the system in the presence of incandescent light

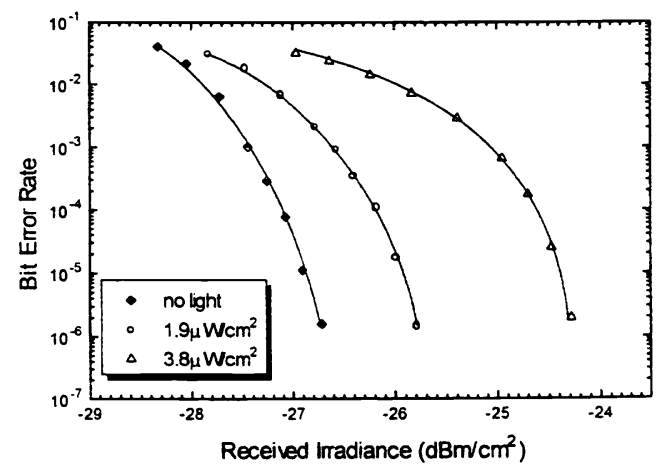

Fig. 10 BER performance of the system in the presence of fluorescent light 